\title{
Consideraciones para el manejo de un implante inmediato en alvéolos infectados: una actualización.
}

\author{
Considerations for the management of an immediate \\ implant in infected sockets: an update.
}

\author{
Britto E Falcón-Guerrero,* Guido S Falcón-Pasapera ${ }^{\ddagger}$
}

\section{RESUMEN}

Introducción: La instalación inmediata del implante posterior a la extracción dental se está convirtiendo en un procedimiento de rutina debido a la reducción del tiempo de tratamiento y a la preservación de las estructuras anatómicas remanentes. Sin embargo, en muchos casos esta técnica involucra dientes afectados con procesos infecciosos. Hasta ahora, no se ha descrito a ciencia cierta el grado de éxito o fracaso del implante inmediato en alvéolos infectados, ni se ha establecido un protocolo para el manejo de este tipo de casos. Objetivo: Realizar una revisión bibliográfica actualizada, para determinar qué consideraciones se deben tener para instalar un implante inmediato en alvéolos con procesos infecciosos. Conclusión: Es posible obtener las ventajas de la instalación inmediata del implante en alvéolos infectados mediante la aplicación de un adecuado manejo clínico, donde se considere un adecuado tratamiento antibiótico, acompañado de un correcto curetaje alveolar, respetar la cadena de asepsia y antisepsia, posición subcrestal del implante y obtener siempre una buena estabilidad primaria del implante.

Palabras clave: Alvéolo dental, infección focal dental, infección, implantes dentales, implante inmediato.

\section{ABSTRACT}

Introduction: The immediate placement of the implant after dental extraction is a procedure that every time is becoming a routine procedure due to the reduction of the treatment time and the preservation of the remaining anatomical structures. However, in many cases, this technique involves affected teeth with infectious processes. Until now, the degree of success or failure of the immediate implant in infected alveoli has not been described for sure; nor has a protocol been established for the management of these types of cases. Objective: Carry out an updated bibliographic review, to determine what considerations must be taken to install an immediate implant in alveoli with infectious processes. Conclusion: Is possible obtain the advantages of the immediate installation of the implant in infected alveoli through the application of adequate clinical management, where appropriate antibiotic treatment is considered, accompanied by proper alveolar curettage, respect the aseptic and antiseptic chain, implant subcrestal position and always obtain a good primary stability of the implant.

Keywords: Tooth socket, dental focal infection, infection, dental implant, immediate implant.

\section{INTRODUCCIÓN}

$\mathrm{L}$ as tasas de éxito a largo plazo para los implantes $\checkmark$ dentales han sido bien documentadas. Sin embargo, para el tratamiento de implantación convencional, la resorción de la cresta alveolar antes de la colocación de los implantes dentales puede afectar la restauración definitiva, especialmente en la zona estética. ${ }^{1}$
Tradicionalmente, antes de colocar los implantes, los alvéolos de extracción dental se dejaban cicatrizar durante varios meses. Sin embargo, muchos pacientes buscan acortar el periodo entre la extracción del diente y la colocación del implante, si es posible instalárselos en la misma sesión, reduciendo el número de intervenciones quirúrgicas, logrando la preservación de la estética y el mantenimiento de las paredes alveolares, siendo acon-

\footnotetext{
* Doctor en Estomatología, Asociación Peruana de Periodoncia y Oseointegración (APPO). Tacna, Perú.

‡ Estudiante, Facultad de Odontología de la Universidad Peruana Cayetano Heredia. Lima, Perú.
} 
sejable colocar el implante en el alvéolo de inmediato, sin esperar la curación. 2,3

Colocar un implante dental después de la extracción del diente se conoce como implantación inmediata. La colocación inmediata del implante está indicada principalmente para reemplazar los dientes faltantes con patologías no susceptibles al tratamiento, tales como dientes con rizólisis de raíz o dientes con lesiones apicales crónicas que no retroceden después del tratamiento endodóntico o cirugía periapical y que no presentan procesos de exacerbación. Además, está indicado en dientes con trauma dentoalveolar y fracturas verticales de la raíz, así como en dientes retenidos. ${ }^{4}$

Las principales ventajas de los implantes inmediatos son la disminución de la resorción del proceso alveolar después de la extracción, se acorta el tiempo de tratamiento y reduce el estrés psicológico del paciente al evitar la necesidad de una segunda etapa quirúrgica. Los implantes inmediatos también permiten la preservación de la morfología de los tejidos duros y blandos, y ayuda a identificar la posición ideal para la colocación del implante. Por otro lado, pueden surgir algunas desventajas, incluida la necesidad de realizar técnicas regenerativas, como los injertos óseos que son tratamientos complicados y costosos. ${ }^{1,4}$

La colocación inmediata de un implante en un alvéolo infectado se ha convertido en un tema muy controvertido. Muchos estudios en animales han demostrado que no hay una diferencia significativa entre las tasas de éxito de colocar inmediatamente un implante en una cavidad infectada, aunque todavía existe controversia en los estudios realizados en humanos. Varios estudios clínicos han indicado que la implantación inmediata en un alvéolo infectado puede aumentar el riesgo de falla del implante dental, mientras que en otros estudios no se observó diferencia en el riesgo del fracaso. ${ }^{2}$

En implantología, un tema que ha despertado un interés particular es la colocación inmediata de implantes dentales en sitios infectados. Por lo que el presente artículo tiene por objetivo realizar una revisión actualizada, para determinar las consideraciones para un procedimiento adecuado al instalar un implante inmediato en alvéolos con procesos de infección latentes.

\section{IMPLANTES INMEDIATOS}

Es la colocación del implante inmediatamente después de la extracción de un diente. Este procedimiento debe combinarse en la mayoría de los pacientes con una técnica de injerto óseo para eliminar los defectos óseos periimplantarios. ${ }^{5}$
La colocación inmediata de un implante dental posterior a la extracción fue propuesta por primera vez por Schulte y Heimke en 1976, y Lazzara y Tolman fueron quienes documentaron su primera aplicación clínica en pacientes. ${ }^{6}$

Las declaraciones de consenso frecuentemente citadas sobre el momento de la colocación del implante definen cuatro categorías según el tiempo transcurrido entre la exodoncia y la implantación: a) colocación inmediata del implante el día de la extracción (tipo 1), b) colocación temprana del implante después de cuatro a ocho semanas de cicatrización de tejidos blandos (tipo 2), c) colocación temprana del implante después de 12-16 semanas de curación parcial del hueso (tipo 3), y d) colocación tardía del implante después de la curación completa del hueso de al menos seis meses (tipo 4). ${ }^{6,7}$

Una reducción del tiempo de tratamiento general en el protocolo de colocación inmediata de implantes representa una solución adecuada. Sin embargo, se cree que la colocación inmediata del implante está significativamente influenciada por la anatomía alveolar local después de la extracción del diente, donde se producen cambios óseos dimensionales y no se controlan mediante la colocación inmediata del implante, lo que puede conducir a resultados estéticos comprometidos a largo plazo. ${ }^{8}$ Esta afectación puede verse influenciada por el grosor del hueso vestibular después de la extracción del diente. Mientras más grueso, conduce a menos alteraciones dimensionales de la cresta alveolar y puede proporcionar resultados más predecibles para la colocación inmediata del implante. 9,10

También se debe tener en cuenta que el recambio óseo durante el periodo de curación puede comprometer la estabilidad del implante y reducir la capacidad de que un implante resista las fuerzas laterales significativas, antes de que se dé una oseointegración adecuada. ${ }^{11}$

Los factores favorables importantes para el éxito de los implantes inmediatos fueron establecidos por Vignoletti y Sanz, exponiendo los siguientes: 1. Espesor sustancial e integridad de las paredes del zócalo; 2 . Posición vertical y horizontal adecuada del implante; 3 . Grosor e integridad gingival; 4. Factores del paciente como la higiene y el tabaquismo. De estos factores, el volumen óseo existente y su capacidad de curación son los más importantes. Cuando no hay suficiente hueso para soportar los implantes o la estructura ósea se resorbe, ocurre la falla del implante. ${ }^{12}$

Testori y colaboradores ${ }^{13}$ reportan requisitos que deben tener los implantes inmediatos exitosos: a) La colocación inmediata de implantes puede ser un procedimiento exitoso en términos de estética, pero es sensible a la técnica y requiere un equipo experimentado. b) La 
colocación inmediata es menos traumática para el paciente, ya que se involucran menos procedimientos quirúrgicos. c) La fase de diagnóstico es de suma importancia, ya que no sólo se abordan las deficiencias del hueso y tejidos blandos, sino también el crecimiento esquelético, los parámetros de los tejidos blandos dentales/implantes (como la erupción pasiva alterada) y la morfología de las raíces adyacentes al área edéntula. d) La carga inmediata posterior a la extracción es factible en sitios infectados. e) La posición correcta del implante debe estar planificada para aceptar un pilar en condiciones adecuadas.

Sin embargo, se debe tener en cuenta que a diferencia de la colocación convencional de implantes, la colocación inmediata de implantes dentales no permite que el organismo controle o maneje la infección residual que pueda quedar dentro de la cavidad de la extracción, antes de colocar el implante inmediato. ${ }^{1}$

\section{IMPLANTES INMEDIATOS EN SITIOS INFECTADOS}

La colocación inmediata de implantes dentales en alvéolos infectados es una alternativa controvertida debido al riesgo de interferencia microbiana con la oseointegración. Muchas veces los dientes con indicación para extracción se ven afectados por procesos infecciosos, sean de origen endodontal y/o periodontal. Se ha reportado que cualquier proceso infeccioso en curso representa una contraindicación para la colocación del implante dental, aumentando el riesgo de falla del implante; estudios anteriores sugieren que la colocación inmediata del implante sólo debe realizarse en alvéolos de extracción libres de infección. Además, se considera que las infecciones bacterianas pueden afectar la oseointegración del implante, conduciendo a un aumento en las tasas de fallas del implante, observándose periimplantitis regresiva por alvéolo infectado. ${ }^{6,14-16}$

Manor y su grupo ${ }^{17}$ encontraron diferentes niveles de contaminación en los sitios de extracción recientes que estaban relacionados con los tipos de infección. Por lo tanto, el control de la contaminación en los sitios de extracción puede ser la clave del éxito de la implantación inmediata en los sitios infectados. Siendo de mucho cuidado que durante la cirugía de implantación, a pesar de realizar una irrigación completa, las bacterias patógenas pueden permanecer y vegetarse en los alvéolos óseos debido a que forman un biofilm autoencapsulado.

Los microrganismos residuales tienen la capacidad de estimular la respuesta inflamatoria, influyendo en la oseointegración ósea y la generación del hueso, lo que causa la falla del implante. Por lo que el sitio infectado debe ser cureteado y desinfectado a fondo para eliminar cualquier tejido de granulación crónica y todos los demás restos de tejido blando, para reducir la actividad inflamatoria. ${ }^{1}$

Se debe tener en cuenta que en los sitios infectados, el hueso vestibular se destruye fácilmente y el tejido blando se hincha o está flácido debido a la falta de grosor de la pared vestibular en comparación con la pared lingual; estos factores pueden aumentar el riesgo de recesión gingival después de la colocación inmediata del implante. Por lo que es recomendable, al momento de realizar la colocación inmediata de implantes en sitios infectados, colocar un injerto de tejido blando para contrarrestar una futura recesión gingival. ${ }^{14}$

Chen y su equipo ${ }^{1}$ determinan en un metaanálisis que la colocación inmediata del implante en sitios infectados y no infectados en la zona estética tenía tasas de supervivencia similares, del mismo modo en los cambios del nivel óseo y cambios en el nivel de la encía. Sin embargo, Zhao y colaboradores ${ }^{2}$ también en un metaanálisis concluyen que colocar inmediatamente un implante dental en un sitio infectado puede aumentar el riesgo de falla de implantes. Sin embargo, mencionan que estos resultados deben interpretarse con precaución.

Pal y su grupo ${ }^{15}$ mencionan que la colocación de un implante inmediato en un alvéolo infectado es una alternativa efectiva y mejora la futura rehabilitación, pero ellos usan plaquetas ricas en factores de crecimiento, para mejorar el resultado del implante dental, coincidiendo con la propuesta de Anitua y colaboradores, ${ }^{16}$ quienes también usan plasma rico en factores de crecimiento al momento de la colocación del implante dental dentro del alvéolo infectado. De manera similar, Medikeri y colegas ${ }^{18}$ aplicaron plaquetas ricas en fibrina en alvéolos frescos contaminados obteniendo una significativa reducción en la resorción ósea y al contrario, lograron conseguir una regeneración ósea más rápida durante la fase inicial postextracción.

Crippa y su equipo ${ }^{19}$ proponen como coadyuvante para el éxito de los implantes inmediatos en alvéolos infectados el uso del láser de erbio-cromo:itrio-escandiogalio-granate (ErCr:YSSG), debido a que éste es capaz de eliminar bacterias de manera más efectiva que los productos químicos.

Lee y su grupo ${ }^{14}$ no encontraron diferencias estadísticamente significativas en las tasas de supervivencia de los implantes inmediatos en los alvéolos de extracción periapicalmente infectados y no infectados, posiblemente debido a la mejora en la fijación del implante; 
y concluyen que los implantes sí se pueden colocar en alvéolos de extracción infectadas, después de realizar un curetaje completo del alvéolo. Y que por estética, se debe considerar siempre el ancho de la encía queratinizada al realizar la colocación inmediata del implante en sitios infectados. Del mismo modo, Zuffetti y colaboradores ${ }^{6}$ encontraron una tasa de supervivencia de los implantes colocados en sitios infectados de $98.4 \%$ que no fue significativamente diferente de los implantes colocados en sitios no infectados $(97.9 \%)$, concluyendo que la colocación inmediata del implante en sitios con infecciones crónicas periodontales o endodontales se puede llevar a cabo de manera segura, siempre que se realice una desinfección alveolar adecuada. ${ }^{8}$

Anitua y su equipo ${ }^{16}$ en su estudio no sólo evalúan la supervivencia de los implantes inmediatos en los alvéolos infectados, sino además evalúan los resultados a largo plazo de la carga inmediata en implantes dentales colocados inmediatamente en alvéolos infectados. Encontrando que la carga inmediata del implante insertado en alvéolos infectados no es un factor de riesgo para la supervivencia del implante, logrando un 93\% de supervivencia en más de cinco años de seguimiento; siempre que se cumpla con el requisito de tener una buena estabilidad primaria inicial (torque $\geq 35 \mathrm{Ncm}$ ). Sin embargo, encontraron una pérdida ósea vestibular en un $25.6 \%$ de casos, que mostraron una pérdida $>1.42 \mathrm{~mm}$, lo que afectaría el resultado estético de los implantes dentales. Por lo que siempre es más que necesario lograr una buena estabilidad primaria para lograr el éxito de los implantes dentales instalados en alvéolos infectados postextracción dental.

Lee y su grupo ${ }^{20}$ investigaron las diferencias histológicas entre implantes inmediatos colocados en sitios con infección crónica y sitios no infectados en un modelo canino. Histológicamente, no se observó inflamación alrededor de los implantes colocados en los alvéolos infectados y no infectados. Concluyendo que la instalación inmediata del implante en un alvéolo infectado no produjo diferencias estadísticamente significativas, en comparación con la colocación en una cavidad ósea no infectada cuando se proporcionó suficiente tiempo de curación.

Pese a estos hallazgos, recientemente Oliveira-Neto y colaboradores ${ }^{21}$ en un metaanálisis concluyen que los implantes dentales inmediatos colocados en sitios infectados presentaron un riesgo estadísticamente más alto de falla que los implantes dentales inmediatos colocados en sitios no infectados. Donde los tejidos pueden mostrar una tasa más alta de mucositis y patología periimplantaria alrededor de los implantes dentales inmediatos colocados en sitios infectados.

\section{CONSIDERACIONES PARA EL MANEJO DE UN IMPLANTE INMEDIATO EN ALVÉOLOS INFECTADOS}

Tomando en cuenta la información actualizada que se ha revisado, se deben tener en cuenta ciertos factores que pueden afectar la tasa de supervivencia de los implantes colocados inmediatamente en los alvéolos infectados, como: la naturaleza de la infección (es decir, de origen endodóncico o periodontal), el protocolo de carga, la integridad de la cresta alveolar, y el uso de materiales regenerativos (como injertos de hueso particulado). ${ }^{6,22}$

Para garantizar un mejor pronóstico posible, se han recomendado varios procedimientos de tratamiento, entre los que se incluyen el desbridamiento y/o curetaje de los alvéolos de extracción, la prescripción de antibióticos sistémicos, el uso de enjuague bucal antiséptico, la aplicación de regeneración tisular guiada o la regeneración ósea guiada, buena estabilidad primaria, y una adecuada restauración inmediata o temprana. 1,6,14,16,20

De manera similar, Alqutaibi ${ }^{23}$ menciona que en la zona estética en los dientes asociados con infección periapical o periodontal, la colocación inmediata de implantes tiende a ser una opción segura y tienen un pronóstico clínicamente aceptable, siempre que se realice un buen plan de tratamiento y se consideren los siguientes requisitos previos: prescripción adecuada de antibióticos sistémicos, uso de un enjuague bucal antes y después de la cirugía, desbridamiento completo de los alvéolos de extracción y un manejo efectivo de la carga de restauraciones provisionales y definitivas. Lo cual coincide con Crippa y colaboradores, ${ }^{19}$ que señalan que las medidas para disminuir la carga bacteriana de los sitios infectados incluyen una limpieza meticulosa, desbridamiento alveolar, la administración de antibióticos y enjuagues bucales con clorhexidina postoperatoria al $0.12 \%$; adicionalmente, ellos sugieren el uso del láser de erbio-cromo:itrio-escandio-galio-granate (ErCr:YSSG) para la descontaminación de los alvéolos infectados después de la extracción. De forma similar, se recomienda el uso de agregados plaquetarios como las plaquetas ricas en fibrina (PRF) que contienen factores de crecimiento con el uso de antibioticoterapia de amplio espectro, pre y postoperatorio, lo que resulta en un mayor porcentaje de supervivencia de los implantes dentales en sitios infectados. ${ }^{18}$

También, se recomienda que en los implantes inmediatos de alvéolos infectados se deben colocar 1 a $2 \mathrm{~mm}$ en posición subcrestal, para prevenir la resorción ósea vestibular. $^{20}$ 
La administración de antibióticos antes de la colocación de un implante en un alvéolo con una infección localizada aumenta el potencial de una oseointegración exitosa del implante; sin embargo, Givens y su grupo ${ }^{24}$ reportaron que la administración profiláctica de antibióticos no parece tener un efecto positivo en las tasas de supervivencia de los implantes colocados en alvéolos infectados; aunque se necesitan más estudios a gran escala para validar estos hallazgos; concluyen que la terapia antibiótica preoperatoria puede no ser necesaria en la terapia de implante inmediato. De manera similar Hosseini y su equipo ${ }^{25}$ encontraron tasas de supervivencia de 100\% (con antibióticos) y de 78\% (sin antibióticos) en implantes inmediatos con procesos infecciosos, y que hubo menor pérdida de tejidos blandos en la parte media vestibular cuando se usaron antibióticos. Sin embargo, no encontraron una diferencia estadísticamente significativa, por lo que los antibióticos parecen tener poco efecto sobre los resultados del tratamiento con implantes inmediatos.

\section{CONCLUSIÓN}

Es posible obtener las ventajas de los implantes inmediatos en alvéolos infectados postextracción dental, mediante la aplicación de un adecuado manejo clínico-quirúrgico, donde se considere un adecuado tratamiento antibiótico, acompañado de un correcto desbridamiento y desinfección alveolar, respetar la cadena de asepsia y antisepsia, posición subcrestal del implante y obtener siempre una buena estabilidad primaria del implante. Algunos autores sugieren que el uso de agregados plaquetarios ricos en factores de crecimiento puede mejorar el pronóstico de esta alternativa de tratamiento.

\section{BIBLIOGRAFÍA}

1. Chen H, Zhang G, Weigl P, Gu X. Immediate placement of dental implants into infected versus noninfected sites in the esthetic zone: a systematic review and meta-analysis. J Prosthet Dent. 2018; 120 (5): 658-667.

2. Zhao D, Wu Y, Xu C, Zhang F. Immediate dental implant placement into infected vs. non-infected sockets: a meta-analysis. Clin Oral Implants Res. 2016; 27 (10): 1290-1296.

3. de Oliveira-Neto OB, Barbosa FT, de Sousa-Rodrigues CF, de Lima FJC. Quality assessment of systematic reviews regarding immediate placement of dental implants into infected sites: an overview. J Prosthet Dent. 2017; 117 (5): 601-605.

4. Jofre J, Valenzuela D, Quintana P, Asenjo-Lobos C. Protocol for Immediate Implant Replacement of Infected Teeth. Implant Dentistry. 2012; 21 (4): 287-294.

5. Laney WR. Glossary of oral and maxillofacial implants. Int J Oral Maxillofac Implants. 2017; 32 (4): Gi-G200.
6. Zuffetti F, Capelli M, Galli F, Del Fabbro M, Testori T. Post-extraction implant placement into infected versus non-infected sites: a multicenter retrospective clinical study. Clin Implant Dent Relat Res. 2017; 19 (5): 833-840.

7. Gallucci GO, Hamilton A, Zhou W, Buser D, Chen S. Implant placement and loading protocols in partially edentulous patients: a systematic review. Clin Oral Implants Res. 2018; 29 Suppl 16: 106-134.

8. Hammerle $\mathrm{CH}$, Chen ST, Wilson TG Jr. Consensus statements and recommended clinical procedures regarding the placement of implants in extraction sockets. Int J Oral Maxillofac Implants. 2004; 19: 26-28.

9. Levine RA, Ganeles J, Gonzaga L, Kan JK, Randel H, Evans CD et al. 10 keys for successful esthetic zone single immediate implants. Compend Contin Educ Dent. 2017; 38 (4): 248-260.

10. Chappuis V, Araujo MG, Buser D. Clinical relevance of dimensional bone and soft tissue alterations post extraction in esthetic sites. Periodontol 2000. 2017; 73 (1): 73-83.

11. Neugebauer J, Traini T, Thams U, Piattelli A, Zoller JE. Periimplant bone organization under immediate loading state. Circularly polarized light analyses: a minipig study. J Periodontol. 2006; 77 (2): 152-160

12. Jensen OT. Dental extraction, immediate placement of dental implants, and immediate function. Oral Maxillofac Surg Clin North Am. 2015; 27 (2): 273-282.

13. Testori T, Weinstein T, Scutellà F, Wang HL, Zucchelli G. Implant placement in the esthetic area: criteria for positioning single and multiple implants. Periodontol 2000. 2018; 77 (1): 176-196.

14. Lee J, Park D, Koo KT, Seol YJ, Lee YM. Comparison of immediate implant placement in infected and non-infected extraction sockets: a systematic review and meta-analysis. Acta Odontol Scand. 2018; 76 (5): 338-345.

15. Pal US, Daga D, Singh RK, Kumar L, Singh M. Comparison of stability with two-time use of platelet-rich growth factor versus one-time use of platelet-rich growth factor in immediate placement of dental implant in infected socket. Natl J Maxillofac Surg. 2018; 9 (2): 209-214.

16. Anitua E, Piñas L, Alkhraisat MH. Long-term outcomes of immediate implant placement into infected sockets in association with immediate loading: a retrospective cohort study. J Periodontol. 2016; 87 (10): 1135-1140.

17. Manor Y, Alkasem A, Mardinger O, Chaushu G, Greenstein RB. Levels of bacterial contamination in fresh extraction sites after a saline rinse. Int J Oral Maxillofac Implants. 2015; 30: 1362-1368.

18. S Medikeri R, Meharwade V, M Wate P, V Lele S. Effect of PRF and allograft use on immediate implants at extraction sockets with periapical infection -clinical and cone beam CT findings. Bull Tokyo Dent Coll. 2018; 59 (2): 97-109.

19. Crippa R, Aiuto R, Guardincerri M, Peñarrocha Diago M, Angiero F. Effect of laser radiation on infected sites for the immediate placement of dental implants. Photobiomodul Photomed Laser Surg. 2020; 38 (3): 186-192.

20. Lee J, Kim S, Koo KT, Seol YJ, Cho HJ, Lee YM. Histologic analyses of immediate implant placement in infected and noninfected sockets: an experimental pilot study in beagle dogs. Int J Oral Maxillofac Implants. 2019; 34 (3): 575-584.

21. de Oliveira-Neto OB, Lemos CA, Barbosa FT, de Sousa-Rodrigues CF, Camello de Lima FJ. Immediate dental implants placed into infected sites present a higher risk of failure than immediate dental implants placed into non-infected sites: systematic review and metaanalysis. Med Oral Patol Oral Cir Bucal. 2019; 24 (4): e518-e528.

22. Calvo-Guirado JL, Romanos GE, Delgado-Ruiz RA. Infected tooth extraction, bone grafting, immediate implant placement and 
immediate temporary crown insertion in a patient with severe type-B hemophilia. BMJ Case Rep. 2019; 12 (3). pii: e229204.

23. Alqutaibi AY. Limited evidence suggests that the immediate placement of dental implants into infected sites versus non-infected sites in the esthetic zone show comparable clinical results. J Evid Based Dent Pract. 2019; 19 (2): 180-182.

24. Givens E Jr, Bencharit S, Byrd WC, Phillips C, Hosseini B, Tyndall D. Immediate placement and provisionalization of implants into sites with periradicular infection with and without antibiotics: an exploratory study. J Oral Implantol. 2015; 41 (3): 299-305.
25. Hosseini B, Byrd WC, Preisser JS, Khan A, Duggan D, Bencharit S. Effects of antibiotics on bone and soft-tissue healing following immediate single-tooth implant placement into sites with apical pathology. J Oral Implantol. 2015; 41 (5): e202-S211.

\section{Correspondencia:}

Britto E Falcón-Guerrero

E-mail: artdent2000@hotmail.com 